\title{
Multiband Ultra-Thin Electromagnetic Band-Gap and Double-Sided Wideband Absorbers Based on Resistive Frequency Selective Surfaces
}

\author{
Xiande Wang ${ }^{*}$, and Douglas H. Werner \\ Department of Electrical Engineering, The Pennsylvania State University
}

\section{Introduction}

Electromagnetic absorbing materials have been extensively studied for use in reducing reflections for a wide variety of applications, ranging from stealth technology to anechoic chambers. One of the most popular techniques is based on a Salisbury screen design, which consists of a resistive sheet placed a quarter-wavelength above a PEC ground plane separated by a dielectric layer [1]. However, for many applications the Salisbury screen is prohibitively thick and a more compact structure is desired. Recently, metamaterial surfaces have been utilized in the design of thin absorber screens [2]. Additionally, they have been utilized in the design of ultra-thin single-bandgap electromagnetic absorbers [3], which were based on using a genetic algorithm to optimize a metallic pattern printed on a very thin dielectric substrate backed by a PEC ground plane. Electromagnetic interference due to multiple reflections also poses a problem for wireless local area networks, and therefore designs that minimize this effect are also of interest. To suppress multiple reflections, a novel absorptive/transmissive FSS was investigated in [4] using a two layer cross dipole FSS screen, while a double-sided permeable wave absorber based on a resistive film design was reported in [5].

Within the realm of metamaterial absorber design, multiband ultra-thin electromagnetic bandgap absorbers and double-sided absorber designs based on resistive frequency selective surfaces (FSSs) have not been widely investigated. In this paper, we present an efficient spectral-domain periodic method of moments (PMM) formulation for the analysis of resistive FSS screens embedded in a stratified medium. We apply a cascading procedure to generate a composite scattering matrix for the entire system consisting of multiple resistive FSS screens embedded in a stratified medium. In addition, the 2-D fast Fourier transform (FFT)-based method was applied to speed up calculation of the required impedance matrix elements. Moreover, an $O(N \log N)$ FFT-based method with 1-D implementation together with the sparse storage technique was employed to accelerate computation of the matrix-vector product within the bi-conjugate gradient (BCG) iterative solver. This hybrid method differs from the 2-D FFT techniques reported in [6]. Finally, this formulation will be applied to the design of multiband ultra-thin electromagnetic bandgap absorbers that operate over a wide range of incidence angles and double-sided wideband absorbers that employ resistive FSS screens.

\section{Resistive FSS Screens Embedded in a Planar Stratified Medium}

\subsection{Sattering from a Single Resistive FSS Screen}

The spectral-domain PMM is employed to analyze a single infinitely thin resistive FSS screen embedded in a stratified medium. Based on Floquet's theorem and impedance boundary conditions, the operator equation which describes surface currents within a unit cell of a FSS screen excited by an incident plane wave can be written as [6]

$$
-\left[\begin{array}{c}
E_{x}^{i n c}(x, y) \\
E_{y}^{i n c}(x, y)
\end{array}\right]=\sum_{m} \sum_{n}\left[\begin{array}{ll}
\tilde{G}_{x x} & \tilde{G}_{x y} \\
\tilde{G}_{y x} & \tilde{G}_{y y}
\end{array}\right]\left[\begin{array}{c}
\tilde{J}_{x}\left(\alpha_{m n}, \beta_{m n}\right) \\
\tilde{J}_{y}\left(\alpha_{m n}, \beta_{m n}\right)
\end{array}\right] \cdot e^{j\left(\alpha_{m n} x+\beta_{m n} y\right)}-R_{s} \cdot\left[\begin{array}{c}
J_{x} \\
J_{y}
\end{array}\right]
$$

where $R_{s}$ is the resistance of the infinitely thin resistive surface (measured in $\Omega /$ square), and $\tilde{G}_{x x}, \tilde{G}_{x y}, \tilde{G}_{y x}$ and $\tilde{G}_{y y}$ are the spectral-domain Green's functions (GFs) for the planar 
stratified medium, which can be derived by the spectral admittance/impedance approach. By expanding the surface current in terms of 2-D roof-top basis functions and then employing Galerkin's procedure, the following linear matrix equation can be obtained:

$$
\left[E^{i n c}\right]=[Z][I]-[F][I],
$$

The sparse impedance matrix, $[F]$, is associated with a sheet impedance, $R_{s}$, and can be quickly calculated due to its simple closed form representation. The 2-D FFT technique can be applied to rapidly calculate the required impedance matrix elements of [Z] [7]. The uniform discretization of the FSS screen and the roof-top basis functions employed here in conjunction with Galerkin's method lead to an impedance matrix [Z] in (2) that has an asymmetric multilevel block-Toeplitz structure for oblique incidence. Hence, an $O(N \log N)$ FFT-based method with a 1-D FFT implementation [8] can also be applied to speed up the matrix-vector product (for $[Z]_{0}[I]$ ) associated within the BCG iterative solver. Note that the matrix-vector product for the portion of $[F] \cdot[I]$ can be rapidly calculated using the sparse storage technique. Upon obtaining the current distribution on the unit cell of the FSS screen, the reflection and transmission coefficients can be calculated for the required harmonic scattered field.

\subsection{Scattering from Multiple Resistive FSS Screens}

In order to analyze multiple resistive FSS screens embedded in a stratified medium, one can use the spectral-domain PMM techniques described in the preceding section. However, by utilizing these techniques, a 2-D FFT-based method cannot be easily applied for fast computation of the required impedance matrix elements of $[Z]$, since the spectral-domain GFs associated with $\left(\rho-\boldsymbol{\rho}^{\prime} ; z-z, z+z^{\prime}\right)$ and $z \neq z^{\prime}$ will require evaluation. To overcome this shortcoming, a more efficient scattering matrix technique was implemented. The technique is based on three steps: (a) multiple resistive FSS structures are first subdivided into subsystems consisting of a single-screen surrounded by a number of dielectric superstrates and substrates; (b) a generalized scattering matrix for each subsystem is individually calculated; (c) the composite scattering matrix for the entire system is then computed by using the formulations given in $[6,7]$ via a cascading procedure. Note that for small problems the LU decomposition solver will be more efficient than the iterative solver to generate the scattering matrix elements.

\section{Numerical Results and Discussion}

The first example will demonstrate the efficiency of the PMM code developed here by analyzing the total CPU time required to perform PMM simulations of a non-resistive FSS screen, which is based on the FSS unit cell illustrated in Fig. 1(b) [9]. In Table 1, data is shown for simulations of the unit cell discretized by a $64 \times 64$ and a $128 \times 128$ grid of pixels. The corresponding CPU time required by the "brute-force" method and the 2-D FFT-based algorithm for nonredundant matrix element filling at a single frequency point are also presented in the table. It can be seen that the BCG iterative solver with the help of the 1-D FFT-based acceleration technique, together with the 2-D FFT-based method for rapid evaluation of impedance matrix elements, provides a faster solver compared to the brute-force method, especially for large problems.

The second example involves a multiband ultra-thin electromagnetic bandgap absorber design. The design consists of a single layer resistive FSS screen with a thin dielectric substrate backed by a PEC ground plane, as shown in Fig. 1(a). The two cases that were investigated are shown in Fig. 2(a) along with their relevant design parameters. These cases involve resistive FSS screen patterns ( $0.5 \Omega /$ square) with identical outer square loop dimensions but different inner square loop dimensions. Plots of the reflection 
coefficient versus frequency reveal two electromagnetic absorbing bands over the range of $1 \mathrm{GHz} \leq f \leq 5 \mathrm{GHz}$ for each configuration (i.e., $1.74 \mathrm{GHz}$ and $3.43 \mathrm{GHz}$ for case 1 and $1.73 \mathrm{GHz}$ and $4.05 \mathrm{GHz}$ for case 2). At the center frequencies of these bands the first case has a thickness of $\lambda_{0} / 68.97$ and $\lambda_{0} / 34.99$, respectively, and the second case has a thickness of $\lambda_{0} / 68.36$ and $\lambda_{0} / 29.63$; where $\lambda_{0}$ corresponds to the wavelength at the center frequency of the absorbing band. The angular performance of the case 1 ultra-thin absorber is illustrated in Fig. 2(b) for both TE and TM wave illumination at $1.73 \mathrm{GHz}$ and $3.43 \mathrm{GHz}$. For the TM illumination at $3.43 \mathrm{GHz}$ the reflection of the absorber is seen to have an attenuation of at least $-20 \mathrm{~dB}$ up to $\theta=50^{\circ}$.

Finally, examples are presented for double-sided absorbers that were designed based on a symmetric configuration, which consists of a two layer resistive FSS screen embedded in a three-layered medium, as shown in Fig. 1(b). The outer dielectric layers are assumed to have identical values for thickness, permittivity, and permeability. Additionally, the same screen patterns are applied to each FSS. The FSS screens have a resistance of $40 \Omega$ /square, while the other relevant parameters used in the simulations are provided in Fig. 3(a). In this figure the reflection and transmission coefficients versus frequency are shown for the two cases with different values of permittivity in the outer dielectric layer. Both cases are seen to exhibit wideband absorbing characteristics. Angular responses at $10.6 \mathrm{GHz}$ and $11.8 \mathrm{GHz}$ are shown in Fig. 3(b) for case 1 and case 2 , respectively. At $11.8 \mathrm{GHz}$ the case 2 design is seen to have a reflection coefficient below $-20 \mathrm{~dB}$ up to an incident angle of $\theta=41^{\circ}$. To improve absorption performance, double-sided absorbers working at two different frequencies for the left and right side, can be constructed by choosing appropriate stratified medium parameters, screen patterns and resistance values. A genetic algorithm optimizer can be applied to this design problem to determine the optimal parameters for enhanced wideband absorption for both polarizations over a wide range of incident angles.

\section{Acknowledgments}

This work was supported in part by the Penn State Materials Research Institute and the Penn State MRSEC under NSF grant DMR-0820404, and by ARO-MURI award 50342-PH-MUR.

\section{References}

[1] R. L. Fante and M.T. McCormack, "Reflection properties of the Salisbury screen," IEEE Trans. Antennas Propagat., vol. 36, pp. 1443-1454, Oct. 1988.

[2] N. Engheta, "Thin absorbing screens using metamaterial surface," IEEE Antennas and Propagat. Soc. Int. Symp., vol. 2, pp. 392-395, June 2002,

[3] D. J. Kern and D. H. Werner, "A genetic algorithm approach to the design of ultra-thin electromagnetic bandgap absorbers," Microwave Opt. Tech. Lett, vol. 38, pp. 61-64, July 2003.

[4] G. I. Kiani, A. R. Weily, and K. P. Esselle, "A novel absorb/transmit FSS for secure indoor wireless networks with reduced multipath fading," IEEE Microw. Wireless Compon. Lett., vol. 16, pp. 378-380, June 2006.

[5] T. Nakamura, C. Dota, and R. Sato, "Double-sided permeable wave absorbers," Electron. Comm. Jpn. (Part I), vol. 90, no. 6, pp. 1-8, 2007.

[6] R. Mittra, C. H. Chan, and T. Cwik, "Techniques for analyzing frequency selective surfaces - a review," IEEE Proc., vol. 76, no. 12, pp. 1593-1614, Dec. 1988.

[7] J. D. Vacchione, "Techniques for analyzing planar, periodic, frequency selective surface systems," Ph.D. Dissertation, University of Illinois at Urbana-Champaign, 1990.

[8] B. E. Barrowes, F. L. Teixeira, and J. A. Kong, "Fast algorithm for matrix-vector multiply of asymmetric multilevel block-Toeplitz matrices in 3-D scattering," Microwave Opt. Tech. Lett., vol. 31, pp. 28-32, 2001.

[9] X. Wang and D. H. Werner, "Accelerated PMM solutions via model-based parameter estimation and fast Fourier transform techniques," IEEE Antennas and Propagat. Soc. Int. Symp., July 5$11,2008$. 
Table 1. Comparison of CPU times for the PMM calculations using different algorithms.

\begin{tabular}{|c|c|c|c|c|}
\hline BCG Iterative Solver Accelerated by the 1-D FFT for the FSS Shown in Fig. 1(b) [9]: $\theta_{i n c}=1^{\circ}, \phi_{i n c}=1^{\circ}$ \\
\hline \multirow{2}{*}{$\begin{array}{c}\text { Filling Impedance } \\
\text { Matrix }\end{array}$} & \multicolumn{2}{|c|}{$\begin{array}{c}\text { Total CPU Time Required for } \\
\text { Both TE and TM Cases } \\
\text { (760 Solutions) }\end{array}$} & \multicolumn{2}{c|}{$\begin{array}{c}\text { CPU Time Required for } \\
\text { Filling Matrix Elements of } O(N) \text { System }\end{array}$} \\
\cline { 2 - 5 } & $64 \times 64$ grid & $128 \times 128$ grid & $64 \times 64$ grid & $128 \times 128$ grid \\
\hline Brute-force Method & $6608.44(\mathrm{sec})$ & $94020.31(\mathrm{sec})$ & $8.063(\mathrm{sec})$ & $125.046(\mathrm{sec})$ \\
\hline 2-D FFT-based Method & $2285.94(\mathrm{sec})$ & $23214.20(\mathrm{sec})$ & $0.141(\mathrm{sec})$ & $0.594(\mathrm{sec})$ \\
\hline
\end{tabular}

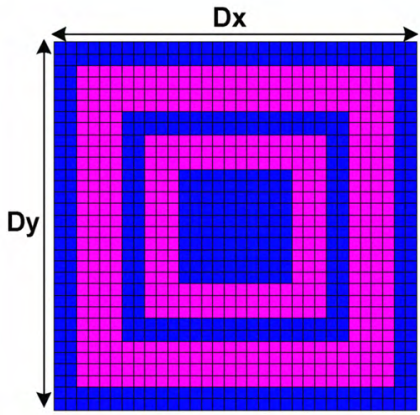

(a)

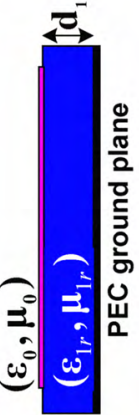

要

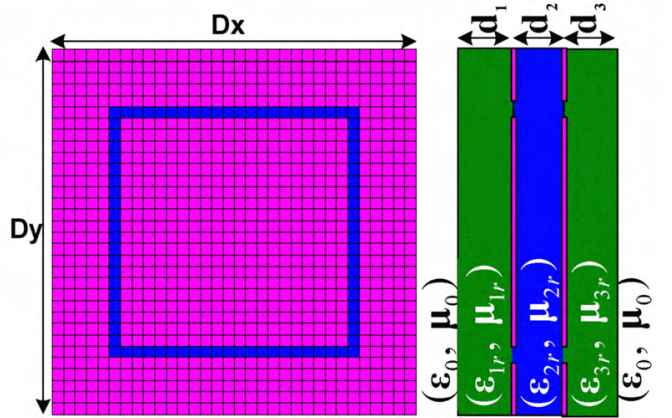

(b)

Fig. 1. (a) Single layer resistive FSS screen backed by a dielectric substrate with a PEC ground plane, (b) Two layer resistive FSS screens embedded in planar stratified medium.
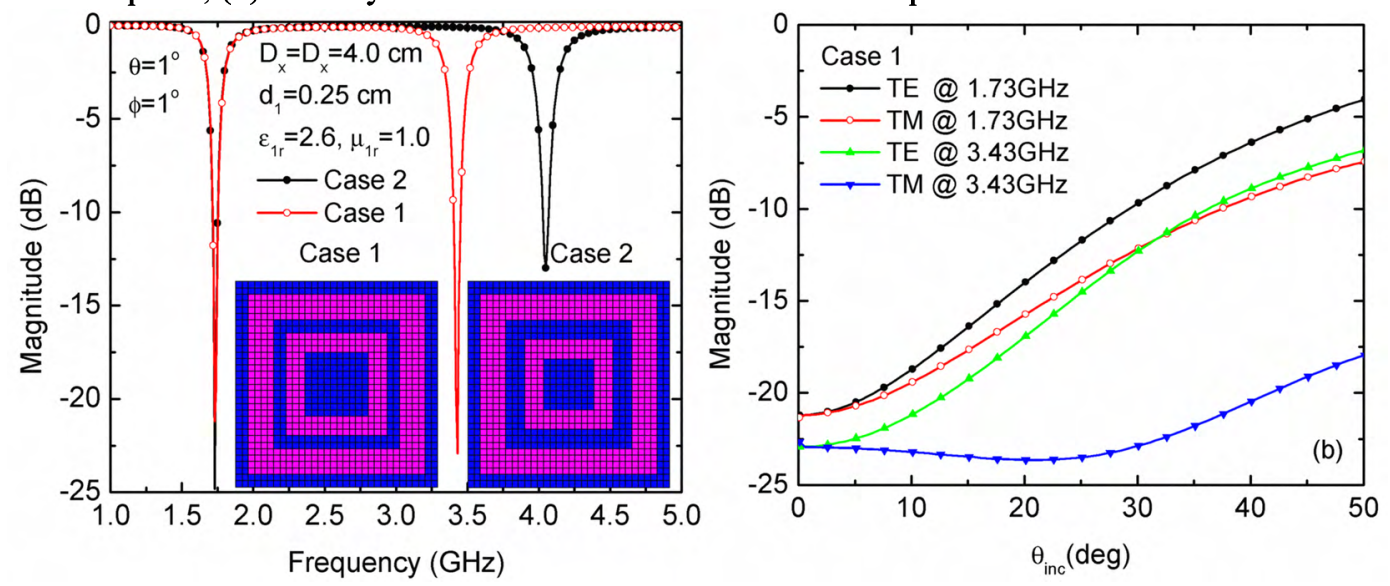

Fig. 2: (a) Reflection coefficient vs. frequency for single-layer resistive FSS absorbers (normal incidence), (b) Angular response for the configuration of case 1 for both TE and TM illumination.
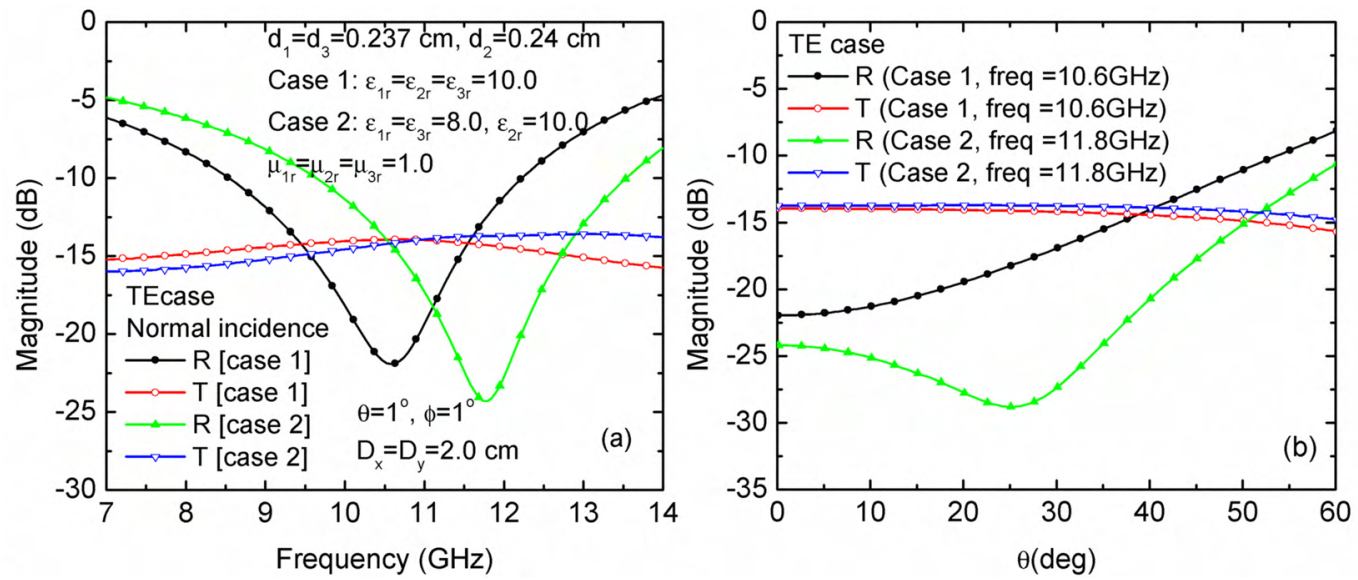

Fig. 3: (a) Reflection and transmission coefficients vs. frequency for the double-sided absorbers,

(b) Angular performance at $10.6 \mathrm{GHz}$ and $11.8 \mathrm{GHz}$ for case 1 and case 2, respectively. 